\title{
Case Report Peritonitis due to Cunninghamella bertholletiae in a patient undergoing continuous ambulatory peritoneal dialysis
}

\author{
Correspondence \\ Jason D. Pimentel \\ pimentel@iprimus.com.au
}

Received 16 June 2005

Accepted 29 August 2005

\author{
Jason D. Pimentel, ${ }^{1}$ Gavin Dreyer ${ }^{2}$ and Gary D. Lum ${ }^{1}$ \\ Northern Territory Government Pathology Service ${ }^{1}$ and Department of Renal Medicine ${ }^{2}$, \\ Royal Darwin Hospital, PO Box 41326, Casuarina, NT 0811, Australia
}

\begin{abstract}
Peritoneal dialysis-associated peritonitis due to fungi of the class Zygomycetes occurs very rarely. A case of fungal continuous ambulatory peritoneal dialysis peritonitis due to Cunninghamella bertholletiae is reported in a 39-year-old Aboriginal woman with end-stage renal failure and diabetes mellitus. This isolate was found to be resistant in vitro to amphotericin B, 5-fluorocytosine, fluconazole, itraconazole, ketoconazole and voriconazole. However, this patient was successfully treated with voriconazole and removal of the Tenckhoff dialysis catheter. Zygomycoses are an emerging threat among immunocompromised patients, including those with chronic renal failure. Zygomycosis due to C. bertholletiae is frequently fatal and is often non-responsive to systemic antifungal therapy. This is believed to be the first reported case of $C$. bertholletiae causing peritonitis in humans and one of the minority of cases involving this organism with a successful outcome.
\end{abstract}

\section{Introduction}

Fungal peritonitis remains a problem in patients undergoing peritoneal dialysis (PD). Peritonitis due to fungi of the class Zygomycetes is very uncommon. We present a case of fungal continuous ambulatory peritoneal dialysis (CAPD) peritonitis in an Aboriginal patient from Darwin, Australia, due to the zygomycete Cunninghamella bertholletiae. To our knowledge this is the first report of this organism causing peritonitis in humans. The patient was treated with voriconazole and made a full recovery. However, the isolate was found to display in vitro resistance to voriconazole. This clinical scenario is followed by a discussion of $C$. bertholletiae and reported strategies for management.

\section{Case report}

On 23 January 2005 a 39-year-old Aboriginal woman presented to the Royal Darwin Hospital with a cloudy PD bag, diarrhoea and vomiting. Her background history included type II diabetes and end-stage renal failure. She had received CAPD for the past 2 years. Blood pressure on presentation was $88 / 60 \mathrm{mmHg}$, serum white cell count (WCC) was $28.2 \times 10^{9} 1^{-1}$ with a neutrophilia of $27 \cdot 4 \times 10^{9} 1^{-1}$. Urea was $12 \cdot 7 \mathrm{mmol} \mathrm{l}^{-1}$ and creatinine was $918 \mu \mathrm{mol} \mathrm{l}^{-1}$. Her temperature was $37 \cdot 4^{\circ} \mathrm{C}$. The PD fluid white cell count was $5600 \times 10^{6} 1^{-1}(92 \%$ neutrophils $)$ and a Gram stain revealed no organisms. She was admitted and started on intra-peritoneal (IP) ceftazidime $1.0 \mathrm{~g}$ daily and cephazolin $1 \cdot 0 \mathrm{~g}$ daily. The initial PD fluid cultures grew one colony of a

Abbreviations: CAPD, continuous ambulatory peritoneal dialysis; FP, fungal peritonitis; IP, intra-peritoneal; PD, peritoneal dialysis.
Pantoea species on a primary culture plate, and therapy was changed to ceftazidime $1 \mathrm{~g}$ IP daily and gentamicin $20 \mathrm{mg}$ IP daily.

During admission several repeat PD fluid specimens were collected. An abdominal CT scan showed free fluid, but no other significant pathology. In spite of IP antibiotic therapy, the clinical picture deteriorated and the PD white cell count remained elevated at $496 \times 10^{6} 1^{-1}$. It was decided to remove the Tenckhoff catheter, which resulted in the resolution of the patient's pain and fever. The catheter, however, was not sent for culture. Maintenance haemodialysis via a native arterio-venous fistula was then commenced.

On 4 February 2005, non-yeast fungal elements were reported in the enrichment culture of PD fluid obtained 2 days earlier. The patient was commenced on voriconazole at $200 \mathrm{mg}$ orally twice daily and followed up as an outpatient over the next 2 weeks. After discharge, her symptoms continued to resolve and her inflammatory markers returned to normal.

Culture of the peritoneal fluid revealed C. bertholletiae. Subsequent fungal susceptibility testing showed in vitro resistance to a wide range of antifungals, including voriconazole. However, due to the excellent clinical response, the patient was maintained on this therapy. After 6 weeks of antifungal therapy, a repeat abdominal CT identified a few small pockets of fluid, one of which was aspirated. The aspirate was clear, did not contain fungal elements and was culture negative. The patient completed a total of 90 days of voriconazole therapy and remained well at 3 month followup. Reinsertion of the PD catheter has been planned. 


\section{Methods}

PD fluid was collected into EDTA tubes (BD Vacutainer, Becton Dickinson) for cell count and sterile containers for Gram's stain and inoculation of primary culture plates. Specimens were also submitted in Aerobic/F and Anaerobic/F BACTEC blood culture bottles (Becton Dickinson) as an enrichment culture. One specimen was submitted in a single Myco/F Lytic bottle (Becton Dickinson). The fluid from EDTA tubes was centrifuged and used to inoculate horse blood agar (HBA) (Oxoid), chocolate blood agar (CBA) (Oxoid) and MacConkey (MAC) (Oxoid) plates as well as a second HBA plate for anaerobic incubation. The BACTEC bottles were placed in a BACTEC 9240 (Becton Dickinson) for incubation. When the BACTEC 9240 detected positive growth, fluid from each bottle was used to inoculate HBA, CBA and MAC plates and another HBA plate for anaerobic incubation. Fluid was obtained from each bottle via a 20-gauge venting needle (BBL, Becton Dickinson). Microscopy was performed with a wet preparation and Gram's staining.

After a fungal isolate was recovered from the enrichment culture, it was subcultured onto Sabouraud agar (SAB) (Oxoid) and incubated at $30{ }^{\circ} \mathrm{C}$ in air. Microscopy of the colonies grown on Sabouraud agar was performed using lacto-phenol cotton blue staining.

\section{Results}

Aside from one colony of a Pantoea species (a probable contaminant), primary culture plates were all culture negative. Additionally, the direct microscopic examination of all PD fluid was negative as well. The initial two BACTEC bottles submitted signalled positive growth in approximately 2 days. However, the wet preparation and Gram's stain from these bottles displayed no organisms. In addition, subcultures from these bottles failed to grow any organisms. A third bottle again had a negative microscopic examination after a positive signal. A close visual inspection of the contents of the bottle revealed clumps of fungal hyphae suspended in the broth. Re-examination of the two bottles submitted earlier showed a similar fungus. Two days later a fourth bottle was found to be positive and fungal colonies were identified in the broth. In total, fungal growth was detected in four separate PD fluid samples.

A wet preparation of the fungal material revealed broad, branching hyphae with very rare septae. On $\mathrm{SAB}$ at $30^{\circ} \mathrm{C}$, the organism grew white fluffy colonies within 3 days and began to turn grey by day 4 . Microscopically, the organism displayed wide and straight sporangiophores, with single pearshaped vesicles bearing oval sporangiola on very short stalks (Fig. 1). The colonial and microscopic morphology along with the speed of growth was consistent with the genus Cunninghamella. This isolate was then referred to the Mycology Unit of the Adelaide Women's and Children's Hospital for identification and antifungal sensitivity testing. Antifungal susceptibilities were determined using broth microdilution. The isolate was confirmed as C. bertholletiae and was found to display in vitro resistance to amphotericin $\mathrm{B}$ $\left(4 \mathrm{ml} \mathrm{l}^{-1}\right)$, 5-fluorocytosine $\left(>64 \mathrm{ml} \mathrm{l}^{-1}\right)$, fluconazole $\left(>256 \mathrm{ml} \mathrm{l}^{-1}\right)$, itraconazole $\left(2 \mathrm{ml} \mathrm{l}^{-1}\right)$, ketoconazole $\left(8 \mathrm{ml} \mathrm{l}^{-1}\right)$ and voriconazole $\left(>16 \mathrm{ml} \mathrm{l}^{-1}\right)$.

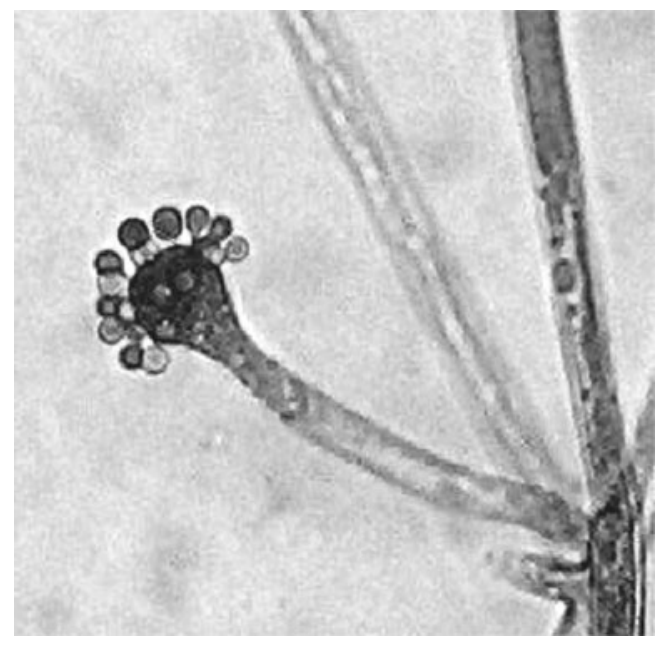

Fig. 1. A sporangiophore of $C$. bertholletiae with a pyriform vesicle bearing immature ellipsoidal sporangioles on sterigmata. Lacto-phenol cotton blue staining; original magnification $\times 400$.

\section{Discussion}

Peritonitis remains a common feature of peritoneal dialysis and complicated the treatment of $64 \%$ of PD patients in a 20 year study by Gloor et al. (2003) This study found the most common aetiology was bacterial, with Staphylococcus epidermidis (30\%) and Staphylococcus aureus (24\%) the most frequently implicated organisms. Catheter replacement or removal was necessary in $63 \%$ of these patients.

Fungal peritonitis (FP) complicates PD less frequently. An 11 year study by Bibashi et al. (2003) reported a rate of $5 \cdot 7 \%$, with a $15 \cdot 9 \%$ associated mortality rate. They found the most common cause of FP was Candida albicans, while filamentous fungi were found much less frequently. The zygomycetes remain an uncommon cause of fungal peritonitis (Nannini et al., 2003). A recent review of seven cases of PD-related zygomycete peritonitis by Nannini et al. (2003) demonstrated a mortality rate of $57 \%$ and that prompt removal of the Tenckhoff catheter was critical for survival. They also noted that most patients were not able to return to PD. Risk factors identified for development of FP include recent bacterial peritonitis, recent exposure to bacterial antibiotics, immunosuppressive therapy and the presence of a bowel perforation (Eisenberg et al., 1986).

C. bertholletiae is a member of the class Zygomycetes in the order Mucorales. The more common pathogens of this order are Rhizopus and Mucor. C. bertholletiae is the only member of the genus identified as a human pathogen and is a resident of soil as well as decaying plant and animal matter (Ribes et al., 2000). It is a rapidly growing organism that initially produces white colonies which usually turn grey with maturity and is thermophilic, growing at $45^{\circ} \mathrm{C}$. Some authors state that a non-pathogenic species, Cunninghamella elegans, is distinguished by purely grey colonies and the absence of growth at $45^{\circ} \mathrm{C}$ (de Hoog et al., 2000). Others consider $C$. 
elegans to be a synonym of $C$. bertholletiae (Richardson et al., 2003). Microscopically, this organism is recognized by the production of sporangiophores that have globose-topyriform vesicles which bear globose-to-ellipsoidal sporangiola on short stalks called sterigmata (Ribes et al., 2000).

C. bertholletiae is not often reported as a human pathogen. Quinio et al. (2004) note that though only 32 cases have been reported, 26 of these have been associated with the death of the patient. Thus it remains a clinically significant pathogen. C. bertholletiae usually acts as an opportunistic pathogen in immunocompromised patients, notably those with haematological malignancies, diabetes mellitus or solid organ transplants (Quinio et al., 2004). This organism usually affects the pulmonary system (Kontoyianis et al., 1994; Mazade et al., 1998); however, its manifestations include sinusitis (Ng et al., 1994), primary cutaneous infection (Quinio et al., 2004), endocarditis (Zhang et al., 2002) and disseminated disease (Kontoyianis et al., 1994; Zhang et al., 2002).

C. bertholletiae is often treated with amphotericin B accompanied by aggressive debridement (Mazade et al., 1998). Published reports give varying results of the success of amphotericin B therapy ( $\mathrm{Ng}$ et al., 1994; Zhang et al., 2002); however, the response to amphotericin B is generally thought to be poor (Ribes et al., 2000). Several reports, including the present case, have documented elevated in vitro MICs to amphotericin B (Mazade et al., 1998; Sun et al., 2002; Dannaoui et al., 2002, 2003). Alternatives to the use of amphotericin B include combination antifungal therapy (Dannaoui et al., 2002) and the use of the new triazole pozaconazole (Sun et al., 2002). Breakthrough infections during voriconazole therapy have been reported with other zygomycoses (Kontoyiannis et al., 2005) and treatment failure has been described (Ritz et al., 2005). The reported in vitro MICs for voriconazole are consistently elevated and the clinical efficacy has not been established (Sun et al., 2002; Dannaoui et al., 2003).

A search of the English literature identified no other cases of peritonitis due to $C$. bertholletiae. Due to its widespread distribution in nature it is often considered a clinical contaminant (Ribes et al., 2000). However, in this case the clinical history and the fact that fungal isolates were found in four separate samples suggest that $C$. bertholletiae was the aetiological agent of this patient's peritonitis. Risk factors included end-stage renal failure, CAPD treatment and diabetes mellitus. The avenue of infection was probably via the Tenckhoff catheter site rather than inhalation, which is the most frequent route. This is supported by the fact that there were no manifestations of dissemination evident in her course or history. Detection of this organism required the use of broth enrichment cultures as all primary culture plates were negative. Though infection by this organism is frequently fatal, our patient experienced a good outcome. The major contribution to her outcome may have been the removal of the PD catheter as this organism displayed in vitro resistance to voriconazole.
In conclusion, we would like to highlight the emerging threat that the zygomycetes represent to patients with an altered immune response. Though this is seen most frequently in those with a compromised immune system secondary to haematological malignancies, it must also be considered in those with chronic renal failure. In the case of $\mathrm{PD}$-associated peritonitis, the practice of $\mathrm{PD}$ catheter removal along with systemic antifungals appears to be the most appropriate therapy. As treatment options for most zygomycetes are generally quite limited, early intervention remains a crucial element for patient survival.

\section{Acknowledgements}

We wish to thank A/Professor David Ellis of the Mycology Unit at the Women's and Children's Hospital, Adelaide, Australia for identifying this isolate and performing susceptibility testing. We also thank $\mathrm{Mr}$ Alan Woodgyer of the University of Melbourne, Microbiological Diagnostic Unit Public Health Laboratory, Melbourne, Australia, for his critical review of this manuscript.

\section{References}

Bibashi, E., Memmos, D., Kokolina, E., Tsakiris, D., Sofianou, D. \& Papadimitriou, M. (2003). Fungal peritonitis complicating peritoneal dialysis during an 11-year period: report of 46 cases. Clin Infect Dis 36, 927-931.

Dannaoui, E., Afeltra, J., Meis, J. F., Verweij, P. E. \& Eurofung Network (2002). In vitro susceptibilities of zygomycetes to combinations of antimicrobial agents. Antimicrob Agents Chemother 46, 2708-2711.

Dannaoui, E., Meletiadis, J., Mouton, J. W., Meis, J. F., Verweij, P. E. \& Eurofung Network (2003). In vitro susceptibilities of zygomycetes to conventional and new antifungals. J Antimicrob Chemother 51, 45-52.

De Hoog, G. S., Guarro, J., Gené, J. \& Figueras, M. J. (2000). Atlas of Clinical Fungi, 2nd edn. Utrecht, Netherlands: Centraalbureau voor Schimmelcultures.

Eisenberg, E. S., Leviton, I. \& Soeiro, R. (1986). Fungal peritonitis in patients receiving peritoneal dialysis: experience with 11 patients and review of the literature. Rev Infect Dis 8, 309-321.

Gloor, H. J., Pandolfi, S. \& Ruttimann, S. (2003). 20 years of peritoneal dialysis in a mid-sized Swiss hospital. Swiss Med Wkly 133, 619-624.

Kontoyianis, D. P., Vartivarian, S., Anaissie, E. J., Samonis, G., Bodey, G. P. \& Rinaldi, M. (1994). Infections due to Cunninghamella bertholletiae in patients with cancer: report of three cases and review. Clin Infect Dis 18, 925-928.

Kontoyiannis, D. P., Lionakis, M. S., Lewis, R. E. \& 8 other authors (2005). Zygomycosis in a tertiary-care cancer center in the era of Aspergillus-active antifungal therapy: a case-control observational study of 27 recent cases. J Infect Dis 191, 1350-1360.

Mazade, M. A., Margolin, J. F., Rossmann, S. N. \& Edwards, M. S. (1998). Survival from pulmonary infection with Cunninghamella bertholletiae: case report and review of the literature. Pediatr Infect Dis J 17, 835-839.

Nannini, E. C., Paphitou, N. I. \& Ostrosky-Zeichner, L. (2003). Peritonitis due to Aspergillus and zygomycetes in patients undergoing peritoneal dialysis: report of 2 cases and review of the literature. Diagn Microbiol Infect Dis 46, 49-54. 
Ng, T. T., Campbell, C. K., Rothera, M., Houghton, J. B., Hughes, D. \& Denning, D. W. (1994). Successful treatment of sinusitis caused by Cunninghamella bertholletiae. Clin Infect Dis 19, 313-316.

Quinio, D., Karam, A., Leroy, J. P., Moal, M. C., Bourbigot, B., Masure, O., Sassolas, B. \& Le Flohic, A. M. (2004). Zygomycosis caused by Cunninghamella bertholletiae in a kidney transplant recipient. Med Mycol 42, 177-180.

Ribes, J. A., Vanover-Sams, C. L. \& Baker, D. J. (2000). Zygomycetes in human disease. Clin Micro Rev 13, 236-301.

Richardson, M. D., Koukila-Kahkola, P. \& Shankland, G. S. (2003). Rhizopus, Rhizomucor, Absidia, and other agents of systemic and subcutaneous zygomycoses. In Manual of Clinical Microbiology, 8th edn, pp. 1761-1780. Edited by P. R. Murray. Washington, DC: American Society for Microbiology.

Ritz, N., Ammann, R. A., Aebischer, C. C., Gugger, M., Jaton, K., Schmid, R. A. \& Aebi, C. (2005). Failure of voriconazole to cure disseminated zygomycosis in an immunocompromised child. Eur J Pediatr 164, 231-235.

Sun, Q. N., Fothergill, A. W., McCarthy, D. I., Rinaldi, M. G. \& Graybill, J. R. (2002). In vitro activities of posaconazole, itraconazole, voriconazole, amphotericin B, and fluconazole against 37 clinical isolates of zygomycetes. Antimicrob Agents Chemother 46, 1581-1582.

Zhang, R., Zhang, J. W. \& Szerlip, H. M. (2002). Endocarditis and hemorrhagic stroke caused by Cunninghamella bertholletiae infection after kidney transplantation. Am J Kidney Dis 40, 842-846. 